\title{
Analysis of AlGaN/GaN high electron mobility transistors with nonalloyed Ohmic contacts achieved by selective area growth using plasma assisted molecular beam epitaxy
}

\author{
Liang Pang, Kyekyoon Kim \\ Department of Electrical and Computer Engineering, \\ University of Illinois at Urbana-Champaign, IL 61801, US
}

\begin{abstract}
We achieved low-resistance nonalloyed Ohmic contacts for $n$-type GaN using selective area growth (SAG) by plasma assisted molecular beam epitaxy (PAMBE). Thus-fabricated high electron mobility transistor (HEMT) demonstrated comparable or more favorable electrical performance than the conventional alloyed counterpart. Meanwhile, the nonalloyed PAMBE-SAG technique avoids problems created by the high temperature annealing, such as the lateral diffusion of contact metals and the generation of surface defects. Consequently, ultrafine electrodes are realized for device downscaling, defect-induced current degradation is alleviated, the reliability and uniformity of HEMTs are improved.
\end{abstract}

Keywords: - AlGaN/GaN HEMT; Nonalloyed Ohmic contacts; Selective area growth; Plasma assisted molecular beam epitaxy; Defects

\section{INTRODUCTION}

GaN-based high electron mobility transistors (HEMTs) have attracted much attention for high power, high frequency, and high temperature applications due to many favorable material properties such as high critical breakdown field and high saturation electron velocity [1-4]. Being a wide bandgap semiconductor however, it is difficult to form low-resistance Ohmic contacts between the contact metals and GaN. Efforts to improve the Ohmic contacts for $n$-type GaN have continued for decades, and Ti/Al-based multiple metallizations with thermal alloying has been adopted as the most successful solution [5-7]. However, the high temperature annealing process takes advantage of the reaction between the metals and $\mathrm{GaN}$ to form metallic TiN, while the residual nitrogen vacancies acting as donors that increases the doping concentration of $n$-GaN under the metal contacts. As a consequence, the accumulation of surface defects created in the process may cause adverse effects such as reliability problem under high temperature operations and current degradation under high voltage $[8,9]$. In addition, the high temperature process causes severe lateral diffusion of the contact metals, hindering further downscaling of GaN-based high-frequency transistors. Last but not least, the surface roughness of the 2-dimensional electron gas (2DEG) due to annealing may give rise to electron mobility degradation and non-uniform current flow. To solve these problems, many nonalloyed techniques have been proposed, such as $\left(\mathrm{NH}_{4}\right)_{2} \mathrm{~S}_{x}$ surface treatment, reactive ion etching, $\mathrm{KrF}$ excimer laser irradiation or selective area growth (SAG) [10-13]. Among them, SAG is the most effective approach to realize low-resistance Ohmic contacts in a damage-free manner, due to the fact that it regrows a highly doped $n$-GaN layer at the contact interface, therefore enhancing the tunneling transport of electrons. While SAG has been achieved by metalorganic chemical vapor deposition (MOCVD) or hydride vapor phase epitaxy (HVPE) [13, 14], plasma assisted molecular beam epitaxy (PAMBE) did not attract much attention due to the lack of growth selectivity, since single-crystalline $\mathrm{GaN}$ grows in the window regions while poly-crystalline $\mathrm{GaN}$ growing on the $\mathrm{SiO}_{2}$ mask as well. However, PAMBE-SAG has its own advantages like ultra-high vacuum and low temperature growth. Particularly, the crystal growth temperature is $300{ }^{\circ} \mathrm{C}$ to $400{ }^{\circ} \mathrm{C}$ lower than the above-mentioned techniques, which is very important to prevent problems such as $\mathrm{GaN}$ film dissociation, Si dopant diffusion, or surface degradation.

In our previous work, PAMBE-SAG was successfully realized, leading to a record low specific contact resistivity of $1.8 \times 10^{-8} \Omega \cdot \mathrm{cm}^{2}$ for an $n$-type GaN channel of metal-semiconductor field-effect transistor (MESFET) [15]. Our technique was also shown to be more effective than ion implantation to improve the Ohmic contact properties $[16,17]$. In this work, the detailed study of the interface between nonalloyed contact metals and the regrown $\mathrm{GaN}$ is performed and compared with the conventional alloyed counterpart using X-ray diffraction (XRD), scanning transmission electron microscopy (STEM), Auger electron microscopy (AES) and scanning electron microscopy (SEM). Electrical performance of both alloyed and nonalloyed HEMTs is also presented herein and discussed. 


\section{EXPERIMENT}

The template used in this work consisted $30 \mathrm{~nm} \mathrm{Al}_{0.15} \mathrm{Ga}_{0.85} \mathrm{~N}$ barrier, $200 \mathrm{~nm}$ GaN channel, $2.5 \mu \mathrm{m}$ semi-insulating GaN, and $30 \mathrm{~nm}$ low-temperature (LT)-GaN buffer, grown on sapphire substrate by MOCVD. For device isolation, $400 \mathrm{~nm} \mathrm{AlGaN} / \mathrm{GaN}$ eplilayers were first etched using an inductively coupled plasma reactive ion etcher (ICP-RIE) with a $\mathrm{Cl}_{2} / \mathrm{Ar}$ plasma. $30 \mathrm{~nm} \mathrm{AlGaN}$ in the drain and source regions was also etched to form the recessed source and drain structure. The SAG process was then conducted as the illustration in Fig. 1. 100-nm-thick $\mathrm{SiO}_{2}$ mask was deposited on the surface using plasma-enhanced chemical vapor deposition (PECVD), and selectively removed by Freon reactive ion etching (RIE) in the drain and source regions to open the contact windows. Before the regrowth process, the sample was thoroughly cleaned by degreaser and acid to remove the native oxide on the surface. A 54-nm-thick single-crystal with doping concentration of $1.0 \times 10^{19} \mathrm{~cm}^{-3}$ was grown in the contact regions. The growth rate was $3.5 \AA / \mathrm{min}$, which was controlled to be slow to obtain high quality $n^{+}-\mathrm{GaN}$ layer. At the same time, polycrystalline GaN was also deposited on the $\mathrm{SiO}_{2}$ mask, which was removed by heated $\mathrm{KOH}(15 \mathrm{wt} \%)$ solution at $75{ }^{\circ} \mathrm{C}$. The residual $\mathrm{SiO}_{2}$ was then etched by buffered oxide etchant (BOE). Before metal deposition, chemical cleaning was carried out using trichloroethylene, acetone, and methanol. Ti/Al/Ti/Au as the contact metals were deposited by electronbeam (E-beam) evaporation on the regrown GaN layers. Finally, Ni/Au were deposited as the Schottky gate contact. Using the same template, conventional alloyed HEMT, for which Ti/Al/Ti/Au metal stack went through rapid thermal annealing (RTA) at $850{ }^{\circ} \mathrm{C}$ for $30 \mathrm{~s}$, was also fabricated. The gate length, width, and source-todrain and gate-to-drain distances were $2 \mu \mathrm{m}, 100 \mu \mathrm{m}, 6 \mu \mathrm{m}$, and $2 \mu \mathrm{m}$, respectively. Micro-structural characteristics of the contact regions were closely examined using Philips X'pert XRD system, JEOL 2010F (S)TEM and PHI 660 Scanning Auger Microprobe. The surface morphology of the contact electrodes was studied using optical microscope and Hitachi S-4800 High Resolution SEM. The electrical characteristics of the HEMTs were measured by an Agilent $4155 \mathrm{C}$ semiconductor parameter analyzer.
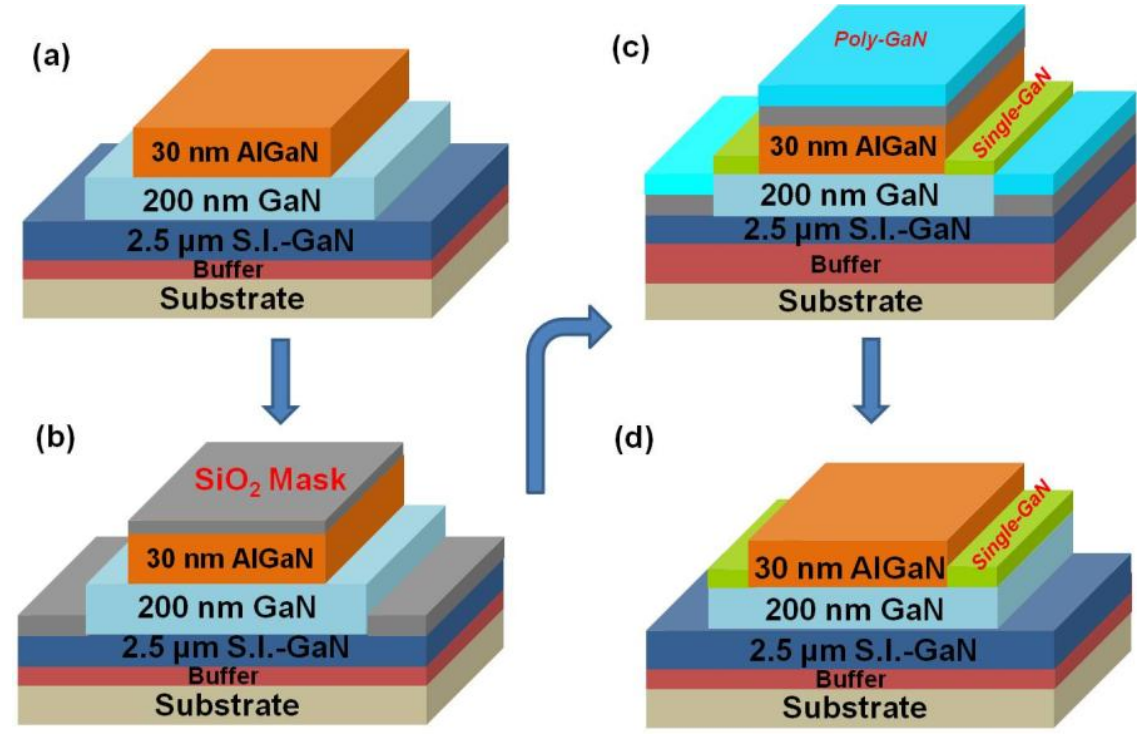

(d)

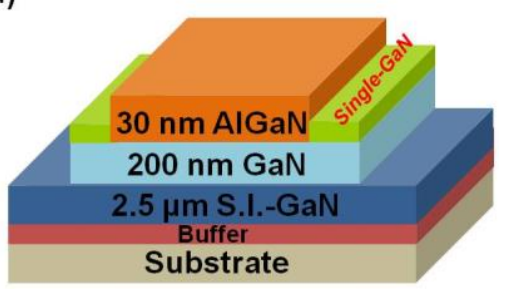

Fig. 1. Schematic illustration of the procedure for selectively regrowing the $n^{+}-\mathrm{GaN}$ layers in the source and drain regions by PAMBE.

\section{RESULTS AND DISCUSSION}

$\mathrm{XRD}$ analysis was carried out to examine the elemental composites at the contact regions. For the nonalloyed sample [Fig. 2(a)], there were several peaks that corresponded to metals such as $\mathrm{Au}, \mathrm{Al}$ and $\mathrm{Ti}$ at $2 \theta$ $=38.3^{\circ}, \mathrm{GaN}(0002)$ and $\mathrm{GaN}(0004)$ at $34.5^{\circ}$ and $72.9^{\circ}$, respectively. There were no other noticeable peaks relating to metal alloys, indicating almost no chemical reaction among the metal stack or at the metalsemiconductor interface. By contrast, for the alloyed sample [Fig. 2(b)], many new compounds such as $\mathrm{Al}_{2} \mathrm{Au}$, $\mathrm{TiAl}$ and $\mathrm{TiN}$, were produced due to the high temperature annealing, indicating severe inter-diffusion and chemical reaction among different elements at the contact regions. Particularly, since TiN was formed by extracting $\mathrm{N}$ atoms from the $\mathrm{GaN}$ layer, the resulting $\mathrm{N}$ vacancies may degrade the epilayer crystallinity and increase the interfacial state density to above $10^{20} \mathrm{~cm}^{-3}$, which may cause reliability problems under high temperature and other harsh conditions [5]. 
(a)

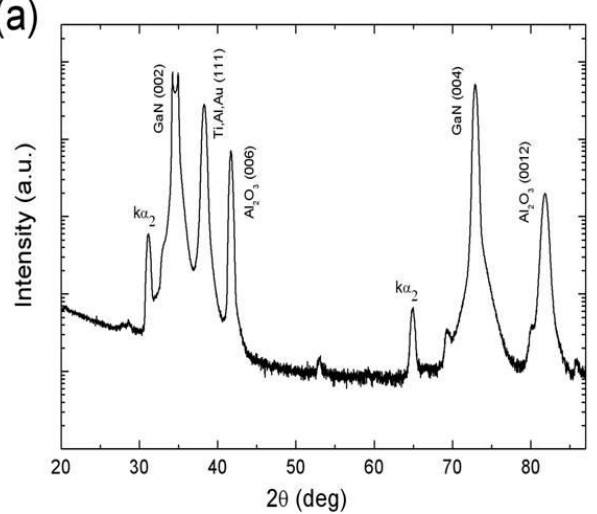

(b)

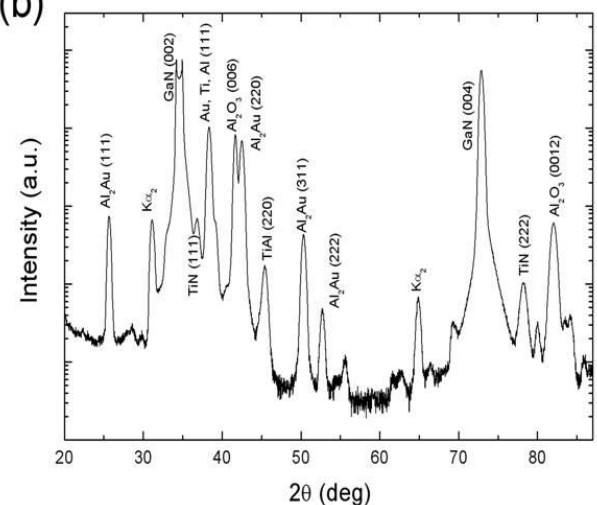

Fig. 2. XRD spectra of the Ti/Al/Ti/Au contacts for (a) the nonalloyed sample and (b) the alloyed sample.

The contact region of the nonalloyed sample was further studied by STEM and AES. From the STEM image in Fig. 3(a), sharp edges between the metals and the regrown GaN layer are observed and no evidence for metal intermixing is found. Fig. 3(b) shows the AES depth profile of $\mathrm{Al}, \mathrm{Ti}, \mathrm{Ga}$ and $\mathrm{Au}$. The result was consistent with the XRD spectra and the STEM profile, which shows that in absence of the annealing process, $\mathrm{Ti}, \mathrm{Al}$ and Au didn't have the chance to diffuse after E-beam evaporation. The interface between Ti and $n^{+}-\mathrm{GaN}$ is also well defined. Such a flat interface with low defect state density may very well enhance the efficiency and uniformity of current transport, while avoiding the reliability problem mentioned above for the alloyed sample.

(a)

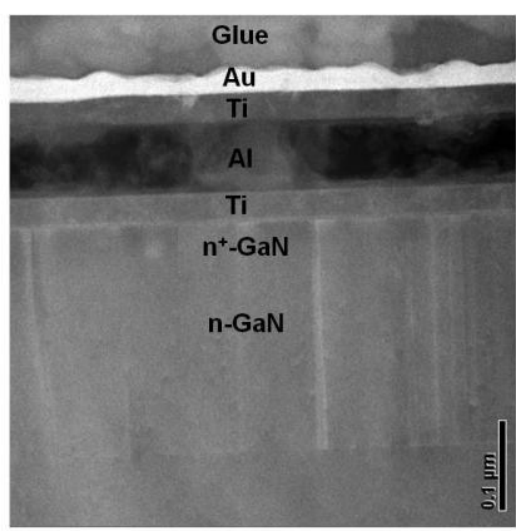

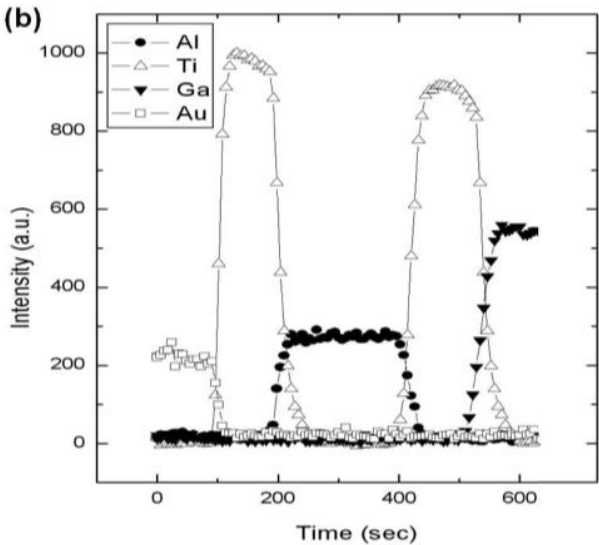

Fig. 3. (a) STEM image and (b) AES depth profile of nonalloyed Ti/Al/Ti/Au contact metals on the regrown $n^{+}$ GaN layer.

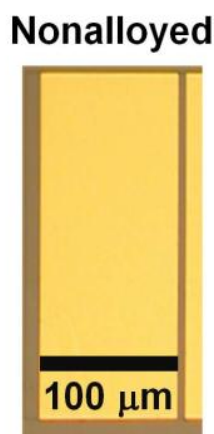

(a)

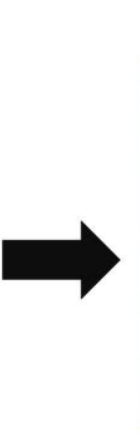

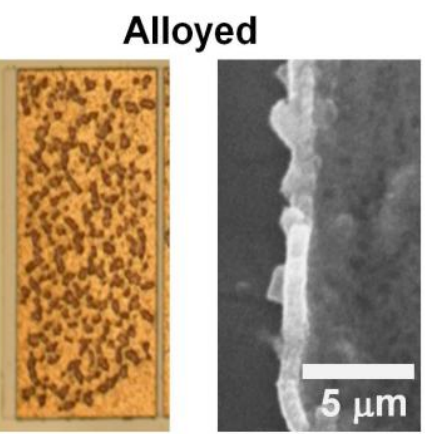

(b) (c)

Fig. 4. (a) Optical image of the nonalloyed contact pad, (b) optical image of the alloyed contact pad, and (c) SEM image of the alloyed contact pad showing the lateral diffusion of metals at the pad edge.

In addition, the nonalloyed process may improve the surface morphology of the contact electrodes. Fig. 4 shows the optical and SEM images of contact pads. It can be seen from Fig. 4(b) that metal alloying induces 
serious surface roughening, which may give rise to non-uniform current flow. Moreover, very severe lateral diffusion of metals is found in Fig. 4(c), making it difficult to accurately define the source, drain and gate electrodes for sub-micron high-frequency HEMTs. The surface and edge roughening is mainly caused by the flow of Al during the high temperature annealing because of its low melting point $\left(\sim 660^{\circ} \mathrm{C}\right)$ [18]. By comparison, the nonalloyed sample in Fig. 4(a) exhibits very sharp and clear edges, making ultrafine electrodes for further device downscaling possible. Therefore, the nonalloyed process should be a good candidate to fabricate sub-micron high-frequency transistors.

For the electrical performance, transmission line method (TLM) was carried out to examine the Ohmic contact resistance. The length and width of the metal pads were $200 \mu \mathrm{m}$ and $100 \mu \mathrm{m}$, and the spacing was varied from 5 to $40 \mu \mathrm{m}$. The current was measured as the voltage swinging from $-1 \mathrm{~V}$ to $+1 \mathrm{~V}$. Three samples were used in the study, naming HEMT-A (no-SAG and no-annealing), HEMT-B (with SAG but no-annealing), and HEMT-C (no-SAG but with annealing). Fig. 5 shows the $I-V$ characteristics of all three samples, measured with a gap spacing of $5 \mu \mathrm{m}$. HEMT-A exhibited a nonlinear $I-V$ curve with low current transport $(I=0.76 \mathrm{~mA}$ at $V=$ $1 \mathrm{~V}$ ), indicating that Ohmic contact was not achieved in this sample due to the energy barrier at the interface between the nonalloyed metals and $\mathrm{GaN}$ epilayer. The regrown $\mathrm{GaN}$ layer by PAMBE can effectively narrow the energy barrier to facilitate electron tunneling. As a result, the current transport is significantly enhanced in HEMT-B $(I=9.5 \mathrm{~mA}$ at $V=1 \mathrm{~V})$, leading to a linear $I-V$ curve and correspondingly Ohmic behavior. By comparison, the current produced by the alloyed contacts in HEMT-C was $11.5 \mathrm{~mA}$ at $1 \mathrm{~V}$. Using the TLM method, the specific contact resistivities for HEMT-B and -C were calculated to be $3.7 \times 10^{-5} \Omega \cdot \mathrm{cm}^{2}$ and $1.5 \times 10^{-5}$ $\Omega \cdot \mathrm{cm}^{2}$, respectively. These results demonstrate that nonalloyed HEMT achieved by PAMBE-SAG could exhibit comparable Ohmic contact properties to the conventional alloyed counterpart, while avoiding problems of the latter associated with the high temperature annealing.

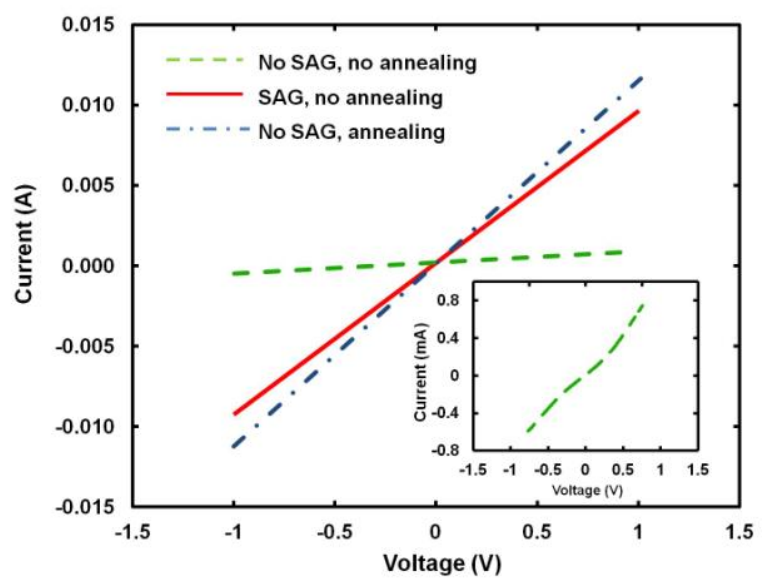

Fig. 5. $I-V$ curves of the TLM pattern with $5 \mu \mathrm{m}$ gap spacing. The inset magnifies the nonlinear $I-V$ curve of HEMT-A.

The $D C$ characteristics of HEMT-B and -C were then measured at room temperature. The $I_{D S}-V_{D S}$ curves were obtained with $V_{D S}$ sweeping from $0 \mathrm{~V} \sim 10 \mathrm{~V}$, while $V_{G S}$ being biased from $1 \mathrm{~V}$ in steps of $-1 \mathrm{~V}$ until the devices were pinched off (Fig. 6). The peak drain current of HEMT-B was $555 \mathrm{~mA} / \mathrm{mm}$ at $V_{G S}=1 \mathrm{~V}$, comparable to $570 \mathrm{~mA} / \mathrm{mm}$ of HEMT-C. Moreover, the drain current degradation at $V_{G S}=10 \mathrm{~V}$ for HEMT-B is only $3.4 \%$, much smaller than that of HEMT-C $(12.3 \%)$. It has been reported that the current degradation is partially caused by the crystallographic-defects, which act as the electron trapping sites [8]. When these defects are charged by the applied drain bias, the 2DEG sheet carriers are depleted, resulting in a decrease in the drain current. Therefore, due to the avoidance of defect generation, the current degradation problem is alleviated in the nonalloyed HEMT. The study of interface state density via pulse measurements is currently ongoing and will be reported elsewhere. 

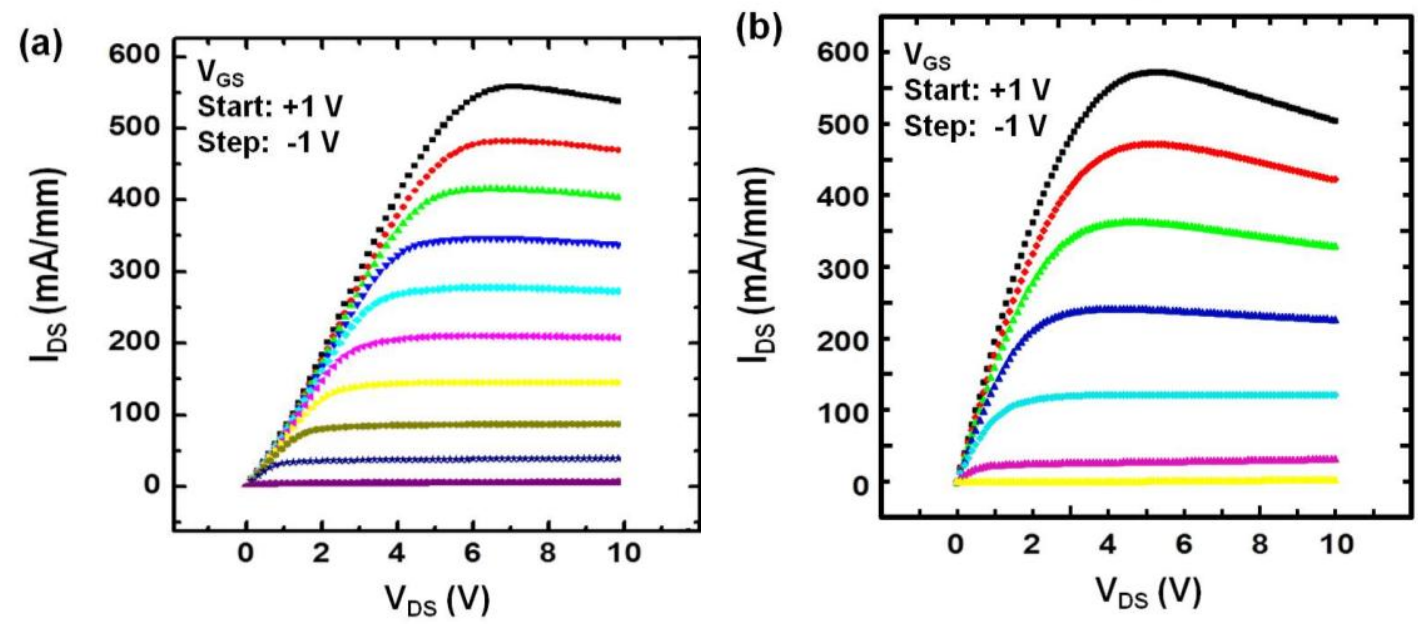

Fig. 6. $I_{D S^{-}} V_{D S}$ characteristics of HEMT-B and HEMT-C.

Last but not least, the Schottky gate leakage current was measured at the reversed gate bias of $-10 \mathrm{~V}$. The leakage currents $\left(I_{\text {gleak }}\right)$ of HEMT-B and HEMT-C were $3.4 \mu \mathrm{A}$ and $18.2 \mu \mathrm{A}$, respectively. $I_{\text {gleak }}$ might be blocked in the nonalloyed HEMT by the epilayer surface discontinuity resulting from the regrown GaN layers. Besides, since defects generally behave as the electron conduction paths, the elimination of surface defects by the nonalloyed technique might also play an important role in suppressing $I_{\text {gleak }}$.

\section{CONCLUSION}

The nonalloyed technique via PAMBE-SAG is demonstrated as an effective approach to realize lowresistance Ohmic contacts for GaN-based HEMTs. It provides comparable contact properties and current carrying capability, as well as lower gate leakage current than the alloyed counterpart, while avoiding problems of the latter caused by the high temperature annealing process, such as lateral diffusion of contact metals and generation of surface defects. Consequently, PAMBE-SAG facilitates further device downscaling, alleviates defect-induced current degradation, and improves the device reliability and uniformity, therefore is considered promising in fabricating GaN-based nonalloyed HEMTs for high power, high frequency, and high temperature applications.

\section{ACKNOWLEDGEMENTS}

This work was supported by Grainger Center for Electric Machinery and Electromechanics of the University of Illinois. The microanalysis was carried out in the Center for Microanalysis of Materials of the University which is partially supported by the U.S. Department of Energy under grant DEF02-91- ER45439.

\section{REFERENCES}

[1] N. Tipirneni, A. Koudymov, V. Adivarahan, J. Yang, G. Simin, and M. A. Khan, The 1.6-kV AlGaN/GaN HFETs, IEEE Electron Device Lett. 27(9), 2006, 716-718.

[2] J. W. Chung, J. Lee, E. L. Piner, and T. Palacios, Seamless on-wafer integration of Si(100) MOSFETs and GaN HEMTs", IEEE Electron Device Lett. 30(10), 2009, 1015-1019.

[3] L. Pang and K. Kim, Bimodal gate-dielectric deposition for improved performance of AlGaN/GaN metal-oxide-semiconductor high-electron-mobility transistors, J. Phys. D: Appl. Phys. 45, 2012, 045105.

[4] L. Pang, Y. G. Lian, D. S. Kim, J. H. Lee, and K. Kim, AlGaN/GaN MOSHEMT With High-Quality Gate-SiO $\mathrm{S}_{2}$ Achieved by Room-Temperature Radio Frequency Magnetron Sputtering, IEEE Trans. Electron Devices, 59(10), 2012, 2650-2655.

[5] D. F. Wang, F. S. Wei, C. Lu, A. Motayed, M. Jah, S. N. Mohammad, K. A. Jones, and L .S. Riba, Lowresistance Ti/Al/Ti/Au multilayer ohmic contact to n-GaN, J. Appl. Phys., 89(11), 2001, 6214-6217.

[6] A. Motayed, R. Bathe, R. M. C. Wood, O. S. Diouf, R. D. Vispute, and S. N. Mohammad, Electrical, thermal, and microstructural characteristics of Ti/Al/Ti/Au multilayer Ohmic contacts to n-type $\mathrm{GaN}, J$. Appl. Phys., 93 (2), 2003, 1087-1094.

[7] Z. F. Fan, S. N. Mohammad, W. Kim, O. Aktas, A. E. Botchkarev, and H. Morkoc, Very low resistance multilayer Ohmic contact to n-GaN, Appl. Phys. Lett., 68, 1996, 1672-1674.

[8] R. Cuerdo, E. Sillero, M. F. Romero, M. J. Uren, M. A. di Forte Poisson, E. Munoz, and F. Calle, HighTemperature Microwave Performance of Submicron AlGaN/GaN HEMTs on SiC, IEEE Electron Device Lett., 30(8), 2009, 808. 
[9] J. Joh and J. A. del Alamo, Critical Voltage for Electrical Degradation of GaN High-Electron Mobility Transistors, IEEE Electron Device Lett., 29(4), 2008, 287-289.

[10] A. Motayed, M. Jah, A. Sharma, W. T. Anderson, C. W. Litton, and S. N. Mohammad, Two-step surface treatment technique: Realization of nonalloyed low-resistance Ti/Al/Ti/Au ohmic contact to n-GaN, $J$. Vac. Sci. Technol. B 22(2), 2004, 663-667.

[11] D. Buttari, A. Chini, G. Meneghesso, E. Zanoni, B. Moran, S. Heikman, N. Q. Zhang, L. Shen, R. Coffie, S. P. DenBaars, and U. K. Mishra, Systematic characterization of $\mathrm{Cl} 2$ reactive ion etching for gate recessing in AlGaN/GaN HEMTs, IEEE Electron Device Lett., 23(3), 2002, 118-120.

[12] H. W. Jang, T. Sands, and J. L. Lee, Effects of KrF Excimer Laser Irradiation on Metal Contacts to ntype and p-type GaN, J. Appl. Phys., 94, 2003, 3529-3535.

[13] S. Heikman, S. Keller, S. P. DenBaars, and U. K. Mishra, Mass transport regrowth of GaN for ohmic contacts to AlGaN/GaN, Appl. Phys. Lett., 78(19), 2001,2876-2878.

[14] K. Hiramatsu, H. Matsushima, T. Shibata, Y. Kawagachi, and N. Sawaki, Selective area growth and epitaxial lateral overgrowth of $\mathrm{GaN}$ by metalorganic vapor phase epitaxy and hydride vapor phase epitaxy, Mater. Sci. Eng. B, 59, 1999, 104-111.

[15] S. J. Hong and K. Kim, Low-resistance Ohmic contacts for high-power GaN field-effect transistors obtained by selective area growth using plasma-assisted molecular beam epitaxy, Appl. Phys. Lett., 89(4), 2006, 042101.

[16] L. Pang, H. C. Seo, P. Chapman, I. Adesida, and K. Kim, Breakdown Voltage Enhancement of AlGaN/GaN High-Electron-Mobility Transistors via Selective-Area Growth for Ohmic Contacts over Ion Implantation, J. Electron. Mater. 39(5), 2010, 499-503.

[17] H. C. Seo, S. Sivaramakrishnan, J. M. Zuo, L. Pang, P. T. Krein, and K. Kim, Interface analysis of $\mathrm{Ti} / \mathrm{Al} / \mathrm{Ti} / \mathrm{Au}$ ohmic contacts with regrown $\mathrm{n}+-\mathrm{GaN}$ layers using molecular beam epitaxy, Surf. and Int. Anal., 43(13), 2010, 1627-1631.

[18] N. Chaturvedi, U. Zeimer, J. Wurfl, and G. Trankle, Mechanism of ohmic contact formation in AlGaN/GaN high electron mobility transistors, Semicond. Sci. Technol., 21, 2006, 175-179. 